\title{
On Torsion-Free Semigroups Generated by Invertible Reversible Mealy Automata
}

\author{
Thibault Godin, Ines Klimann, and Matthieu Picantin ${ }^{\star}$ \\ Univ Paris Diderot, Sorbonne Paris Cité, LIAFA, UMR 7089 CNRS, Paris France \\ \{godin,klimann, picantin\}@liafa.univ-paris-diderot.fr
}

\begin{abstract}
This paper addresses the torsion problem for a class of automaton semigroups, defined as semigroups of transformations induced by Mealy automata, aka letter-by-letter transducers with the same input and output alphabet. The torsion problem is undecidable for automaton semigroups in general, but is known to be solvable within the well-studied class of (semi)groups generated by invertible bounded Mealy automata. We focus on the somehow antipodal class of invertible reversible Mealy automata and prove that for a wide subclass the generated semigroup is torsion-free.
\end{abstract}

Keywords: automaton semigroup, reversible Mealy automaton, labeled orbit tree, torsion-free semigroup

\section{Introduction}

In this paper we address the torsion problem for a class of automaton semigroups. In a (semi)group, a torsion - or periodic - element is an element of finite order, that is an element generating a finite monogenic sub(semi)group. In particular, a (semi)group is torsion-free (resp. torsion) if its only torsion element is its possible identity element (resp. if all its elements are torsion elements). Like most of the major group or semigroup theoretical decision problems, the word, torsion and finiteness problems are undecidable in general [8].

Automaton (semi)groups, that is (semi)groups generated by Mealy automata, were formally introduced a half century ago (for details, see [9] and references therein). Two decades later, important results started revealing their full potential. In particular, contributing to the so-called Burnside problem, the articles 215] construct particularly simple Mealy automata generating infinite finitely generated torsion groups, and, answering the so-called Milnor problem, the articles 616] describe Mealy automata generating the first examples of (semi)groups with intermediate growth. Since these pioneering works, a substantial theory continues to develop using various methods, ranging from finite automata theory to geometric group theory and never ceases to show that automaton (semi)groups possess multiple interesting and sometimes unusual features.

\footnotetext{
* The authors are partially supported by the French Agence Nationale pour la Recherche, through the Project MealyM ANR-JCJC-12-JS02-012-01.
} 
For automaton (semi)groups, the word problem is solvable using standard minimization techniques [7]12]19. The torsion problem and the finiteness problem for automaton semigroups have been proven to be undecidable 14 but remain open for automaton groups. However there exist various criteria for recognizing whether such a (semi)group or one of its element has finite order, see for instance [13]4|9]10]11|18|20|22|23|25|27|28. In particular, there are many partial methods to find elements of infinite order in such (semi)groups. Their efficiency may vary significantly. By contrast, the class of so-called invertible bounded Mealy automata, which has received considerable attention, admits an effective solution to both conjugacy and order problems $[5] 10[27$. This class happens to correspond to some tight restriction on the underlying automata: the non-trivial cycles are disjoint and none can be reached from another.

Here we tackle the torsion problem, focusing on a very different class of Mealy automata, namely reversible Mealy automata, in which each connected component turns out to be strongly connected. This class was known as the class for which most of the existing partial methods do not work or perform poorly. We prove that for a wide subclass of invertible reversible Mealy automata - roughly the non-bireversible ones - the generated semigroup is torsion-free. It is worth mentioning that the class of bounded Mealy automata and the class of reversible Mealy automata are somehow at the opposite ends of the spectrum.

The proof of torsion-freeness relies on deep structural properties of the so-called labeled orbit tree which happens to capture the behavior of the (strongly) connected components during the exponentiation of a reversible Mealy automaton, and it gives hopefully a new insight even in the still mysterious subclass of bireversible Mealy automata (see 720/24 and the references therein).

The paper is organized as follows. In Section 2 we set up notation, provide well-known definitions and facts concerning Mealy automata and automaton semigroups. Some results concerning connected components of reversible Mealy automata are given in Section 3 . In Section 4 we introduce a crucial construction, namely the labeled orbit tree of a Mealy automaton, and define the notion of a self-liftable path, especially relevant for investigating torsion-freeness. Finally, Section 5 contains the proof of our main result.

\section{Mealy Automata}

We first recall the formal definition of an automaton. A (finite, deterministic, and complete) automaton is a triple $\left(Q, \Sigma, \delta=\left(\delta_{i}: Q \rightarrow Q\right)_{i \in \Sigma}\right)$, where the stateset $Q$ and the alphabet $\Sigma$ are non-empty finite sets, and where the $\delta_{i}$ are functions.

A Mealy automaton is a quadruple $\left(Q, \Sigma, \delta=\left(\delta_{i}: Q \rightarrow Q\right)_{i \in \Sigma}, \rho=\left(\rho_{x}: \Sigma \rightarrow\right.\right.$ $\left.\Sigma)_{x \in Q}\right)$, such that both $(Q, \Sigma, \delta)$ and $(\Sigma, Q, \rho)$ are automata. In other terms, a Mealy automaton is a complete, deterministic, letter-to-letter transducer with the same input and output alphabet. 

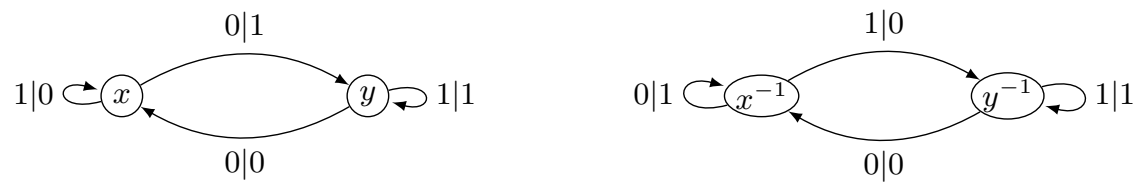

Fig. 1: An invertible reversible non-bireversible Mealy automaton $\mathcal{L}$ (left) and its inverse $\mathcal{L}^{-1}$ (right), both generating the lamplighter group $\mathbb{Z}_{2}$ 2 $\mathbb{Z}$ (see [17]).

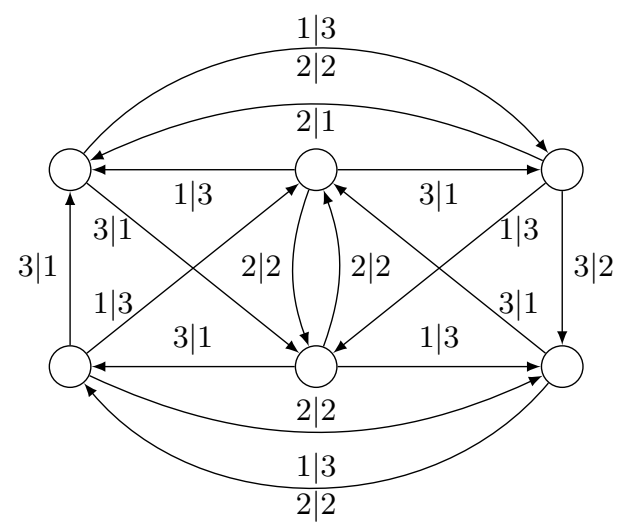

Fig. 2: A 3-letter 6-state inv. reversible non-bireversible Mealy automaton $\mathcal{J}$.

The graphical representation of a Mealy automaton is standard, see Figures 1 and 2 ,

In a Mealy automaton $\mathcal{A}=(Q, \Sigma, \delta, \rho)$, the sets $Q$ and $\Sigma$ play dual roles. So we may consider the dual (Mealy) automaton defined by $\mathfrak{d}(\mathcal{A})=(\Sigma, Q, \rho, \delta)$. Alternatively, we can define the dual Mealy automaton via the set of its transitions:

$$
x \stackrel{i \mid j}{\longrightarrow} y \in \mathcal{A} \quad \Longleftrightarrow \quad i \stackrel{x \mid y}{\longrightarrow} j \in \mathfrak{d}(\mathcal{A}) .
$$

Definition 1. A Mealy automaton $(Q, \Sigma, \delta, \rho)$ is said to be invertible if the functions $\left(\rho_{x}\right)_{x \in Q}$ are permutations of $\Sigma$ and reversible if the functions $\left(\delta_{i}\right)_{i \in \Sigma}$ are permutations of $Q$.

Consider a Mealy automaton $\mathcal{A}=(Q, \Sigma, \delta, \rho)$. Let $Q^{-1}=\left\{x^{-1}, x \in Q\right\}$ be a disjoint copy of $Q$. The inverse $\mathcal{A}^{-1}$ of $\mathcal{A}$ is defined by the set of its transitions:

$$
x \stackrel{i \mid j}{\longrightarrow} y \in \mathcal{A} \quad \Longleftrightarrow \quad x^{-1} \stackrel{j \mid i}{\longrightarrow} y^{-1} \in \mathcal{A}^{-1} .
$$

If $\mathcal{A}$ is invertible, then its inverse $\mathcal{A}^{-1}$ is a Mealy automaton, see for instance Figure 1.

Definition 2. A Mealy automaton is bireversible if it is invertible, reversible and its inverse is reversible. 
The terms "invertible", "reversible", and "bireversible" are standard since [21]. Figure 3 gives characterizations of invertibility and reversibility in terms of forbidden configurations in a Mealy automaton.

Here we define a new class:

Definition 3. A Mealy automaton is coreversible whenever Configuration (c) in Figure 3 does not occur. This means that each output letter induces a permutation on the stateset.

The bireversible Mealy automata are those which are simultaneously invertible, reversible, and coreversible. We emphasize that an invertible reversible Mealy automaton is bireversible if and only if it is coreversible.

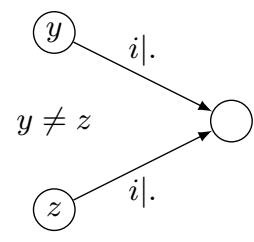

(a)

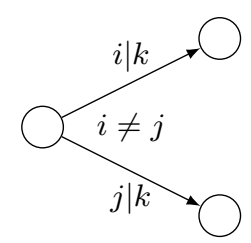

(b)

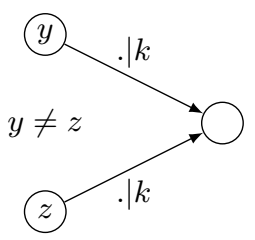

(c)

Fig. 3: Configuration (a) is forbidden for reversible automata, Configuration (b) for invertible ones, and Configuration (c) for coreversible ones.

We view $\mathcal{A}=(Q, \Sigma, \delta, \rho)$ as an automaton with an input and an output tape, thus defining mappings from input words over $\Sigma$ to output words over $\Sigma$. Formally, for $x \in Q$, the map $\rho_{x}: \Sigma^{*} \rightarrow \Sigma^{*}$, extending $\rho_{x}: \Sigma \rightarrow \Sigma$, is defined recursively by:

$$
\forall i \in \Sigma, \forall \mathbf{s} \in \Sigma^{*}, \quad \rho_{x}(i \mathbf{s})=\rho_{x}(i) \rho_{\delta_{i}(x)}(\mathbf{s})
$$

Equation (11) can be easier to understood if depicted by a cross-diagram (see [1]):

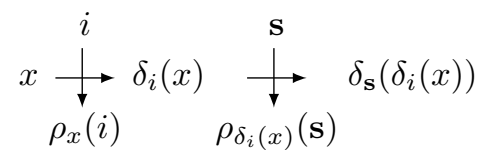

By convention, the image of the empty word is itself. The mapping $\rho_{x}$ for each $x \in Q$ is length-preserving and prefix-preserving. We say that $\rho_{x}$ is the production function associated with $(\mathcal{A}, x)$. For $\mathbf{x}=x_{1} \cdots x_{n} \in Q^{n}$ with $n>0$, set $\rho_{\mathbf{x}}: \Sigma^{*} \rightarrow \Sigma^{*}, \rho_{\mathbf{x}}=\rho_{x_{n}} \circ \cdots \circ \rho_{x_{1}}$. Denote dually by $\delta_{i}: Q^{*} \rightarrow Q^{*}, i \in \Sigma$, the production functions associated with the dual automaton $\mathfrak{d}(\mathcal{A})$. For $\mathbf{s}=$ $s_{1} \cdots s_{n} \in \Sigma^{n}$ with $n>0$, set $\delta_{\mathbf{s}}: Q^{*} \rightarrow Q^{*}, \delta_{\mathbf{s}}=\delta_{s_{n}} \circ \cdots \circ \delta_{s_{1}}$.

The semigroup of mappings from $\Sigma^{*}$ to $\Sigma^{*}$ generated by $\left\{\rho_{x}, x \in Q\right\}$ is called the semigroup generated by $\mathcal{A}$ and is denoted by $\langle\mathcal{A}\rangle_{+}$. When $\mathcal{A}$ is invertible, its 
production functions are permutations on words of the same length and thus we may consider the group of mappings from $\Sigma^{*}$ to $\Sigma^{*}$ generated by $\left\{\rho_{x}, x \in Q\right\}$. This group is called the group generated by $\mathcal{A}$ and is denoted by $\langle\mathcal{A}\rangle$.

It is know from [1] that the possible behaviors of invertible reversible nonbireversible Mealy automata provide less variety than those of bireversible automata whenever finiteness is concerned:

Proposition 4. ([1, Corollary 22]) Any invertible reversible non-bireversible Mealy automaton generates an infinite group.

Note that the ratio of these invertible reversible non-bireversible Mealy automata tends to supersede the bireversible one, when the size of alphabet and/or stateset increases.

\section{On the Behavior of Connected Components}

In this section, we gather some properties satisfied by the connected components of the underlying graph of a reversible Mealy automaton and we focus on those properties preserved when making products. We use the following crucial property: any connected component of a reversible Mealy automaton is strongly connected. Our main tool, described in the next section, captures the behavior of the connected components of the successive powers of a given reversible Mealy automaton, allowing a much finer analysis.

Definition 5. Let $\mathcal{A}=(Q, \Sigma, \delta, \rho)$ and $\mathcal{B}=\left(Q^{\prime}, \Sigma, \delta^{\prime}, \rho^{\prime}\right)$ be two Mealy automata acting on the same alphabet. Their product is the Mealy automaton $\mathcal{A} \times \mathcal{B}=\left(Q \times Q^{\prime}, \Sigma, \gamma, \pi\right)$ with transition

$$
x y \stackrel{i \mid \rho_{y}^{\prime}\left(\rho_{x}(i)\right)}{\longrightarrow} \delta_{i}(x) \delta_{\rho_{x}(i)}^{\prime}(y),
$$

which can be seen in terms of cross-diagram as:

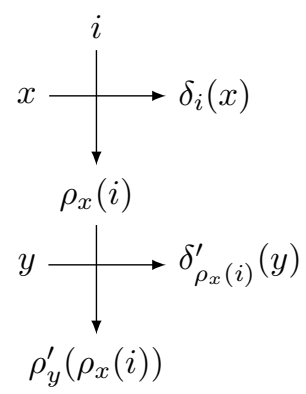

Note that the product of two reversible (resp. invertible) Mealy automata is still a reversible (resp. invertible) Mealy automaton. Let us consider the coreversibility property. 
Lemma 6. Let $\mathcal{A}$ and $\mathcal{B}$ be Mealy automata on the same alphabet with $\mathcal{A}$ connected and reversible. Then, for any connected component $\mathcal{C}$ of $\mathcal{A} \times \mathcal{B}$, every state of $\mathcal{A}$ occurs as a prefix of some state of $\mathcal{C}$.

Proof. Let $\mathcal{A}=(Q, \Sigma, \delta, \rho)$ and let $\mathcal{C}$ be a connected component of $\mathcal{A} \times \mathcal{B}$. Let $x x^{\prime} \in \mathcal{C}$ and $y \in Q$. Since $\mathcal{A}$ is connected and reversible, there exists $\mathbf{s} \in \Sigma^{*}$ satisfying $y=\delta_{\mathbf{s}}(x)$, hence $y$ is a prefix of the state $\delta_{\mathbf{s}}\left(x x^{\prime}\right)$ in $\mathcal{C}$.

Proposition 7. Let $\mathcal{A}$ and $\mathcal{B}$ be reversible Mealy automata on the same alphabet. If $\mathcal{A}$ is connected and non-coreversible, then every connected component of $\mathcal{A} \times \mathcal{B}$ is reversible and non-coreversible.

Proof. Let $Q$ be the stateset of $\mathcal{A}$ and let $\mathcal{C}$ be a connected component of $\mathcal{A} \times \mathcal{B}$. As $\mathcal{A}$ and $\mathcal{B}$ are reversible, so is $\mathcal{C}$.

Since $\mathcal{A}$ is not coreversible, there exist two states $x \neq y \in Q$ leading to the same state $z$, when producing the same letter $j$ :

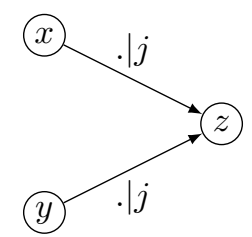

that is,

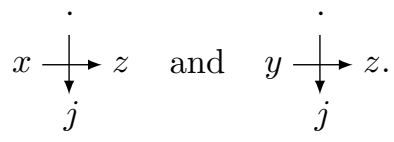

By Lemma 6, $\mathcal{C}$ admits a state prefixed with $x$, say $x x^{\prime}$. Let

$$
x^{\prime} \underset{\stackrel{k}{\vec{k}}}{\stackrel{j}{\longrightarrow}} z^{\prime}
$$

be a transition of $\mathcal{B}$, then the following configuration occurs in $\mathcal{C}$ :
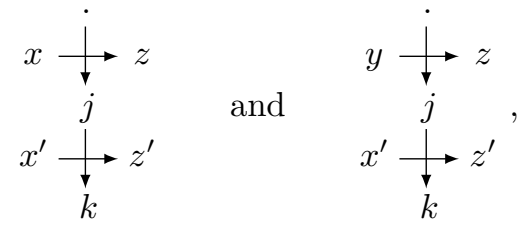

that is,

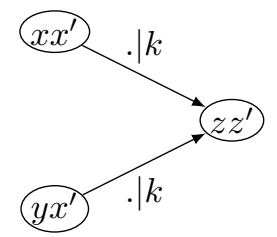

which means that $\mathcal{C}$ cannot be coreversible.

A convenient and natural operation is to raise a Mealy automaton to some power. The $n$-th power of $\mathcal{A}=(Q, \Sigma, \delta, \rho)$ is recursively defined. By convention, $\mathcal{A}^{0}$ is the trivial Mealy automaton with only one state, which acts like identity on $\Sigma$. For $n>0, \mathcal{A}^{n}$ is the Mealy automaton

$$
\mathcal{A}^{n}=\left(Q^{n}, \Sigma,\left(\delta_{i}: Q^{n} \rightarrow Q^{n}\right)_{i \in \Sigma},\left(\rho_{\mathbf{u}}: \Sigma \rightarrow \Sigma\right)_{\mathbf{u} \in Q^{n}}\right)
$$


Corollary 8. If a Mealy automaton is (invertible) reversible without coreversible connected component, then every connected component of any of its powers is (invertible) reversible and non-coreversible.

Definition 9. 1724 The action of a Mealy automaton $\mathcal{A}$ is said to be spherically transitive or level-transitive whenever all the powers of $\mathfrak{d}(\mathcal{A})$ are connected.

\section{The Labeled Orbit Tree}

There exist strong links between the successive sizes of the connected components of the powers of a reversible Mealy automaton and some finiteness properties of the generated semigroup, as emphasized by the two following results. Such links can be captured by a suitable tree, playing a fundamental role in the sequel.

Proposition 10. A reversible Mealy automaton generates a finite semigroup if and only if the sizes of the connected components of its powers are bounded.

The latter is proven in [20] within the framework of invertible reversible Mealy automata, but the invertibility is not invoked in the proof. We need the following result, also from [20].

Proposition 11. Let $\mathcal{A}=(Q, \Sigma, \delta, \rho)$ be an invertible reversible Mealy automaton. For any $\mathbf{u} \in Q^{+}$, the following are equivalent:

(i) the action $\rho_{\mathbf{u}}$ induced by $\mathbf{u}$ has finite order;

(ii) the sizes of the connected components of $\left(\mathbf{u}^{n}\right)_{n \in \mathbb{N}}$ are bounded.

A direct consequence of Proposition 10 provides a simple yet interesting result concerning torsion-freeness.

Corollary 12. Let $\mathcal{A}$ be a reversible Mealy automaton. Whenever the action of $\mathfrak{d}(\mathcal{A})$ is spherically transitive, the semigroup $\langle\mathcal{A}\rangle_{+}$is torsion-free.

Proof. Let $\mathcal{A}$ be a Mealy automaton with stateset $Q$ such that all its powers are connected. By Proposition 10, $\mathcal{A}$ generates an infinite semigroup.

Assume that there exists $\mathbf{u} \in Q^{+}$whose action has finite order, say $\rho_{\mathbf{u}^{p}}=\rho_{\mathbf{u}^{q}}$ with $p<q$. By reversibility of $\mathcal{A}$, every state of $\mathcal{A}^{q}$ is equivalent to some state of $\mathcal{A}^{p}$, hence $\mathcal{A}$ generates a finite semigroup, which is a contradiction.

Corollary 12 applies for instance to the Mealy automaton $\mathcal{L}$ on Figure 1(left): the subsemigroup of the lamplighter group generated by $x$ and $y$ is torsion-free.

We are now ready to introduce our main tool.

Definition 13. Let $\mathcal{A}$ be a reversible Mealy automaton with stateset $Q$. Rooted in $\mathcal{A}^{0}$, the labeled orbit tree $\mathfrak{t}(\mathcal{A})$ is constructed as the graph of the (strongly) connected components of the powers of $\mathcal{A}$, with an edge between two nodes $\mathcal{C}, \mathcal{D}$ whenever there is $\mathbf{u} \in \mathcal{C}$ with $\mathbf{u} x \in \mathcal{D}$ and $x \in Q$, such an edge being labeled by the (integer) ratio $|\mathcal{D}| /|\mathcal{C}|$. 
Such a tree $\mathfrak{t}(\mathcal{A})$ is more precisely named the labeled orbit tree of the dual $\mathfrak{d}(\mathcal{A})$ since it can be seen as the tree of the orbits of $Q^{*}$ under the action of the $\operatorname{group}\langle\mathfrak{d}(\mathcal{A})\rangle$ (see [13/20]).

Figure 4 displays the labeled orbit tree $\mathfrak{t}(\mathcal{J})$, where $\mathcal{J}$ is the Mealy automaton defined in Figure 2

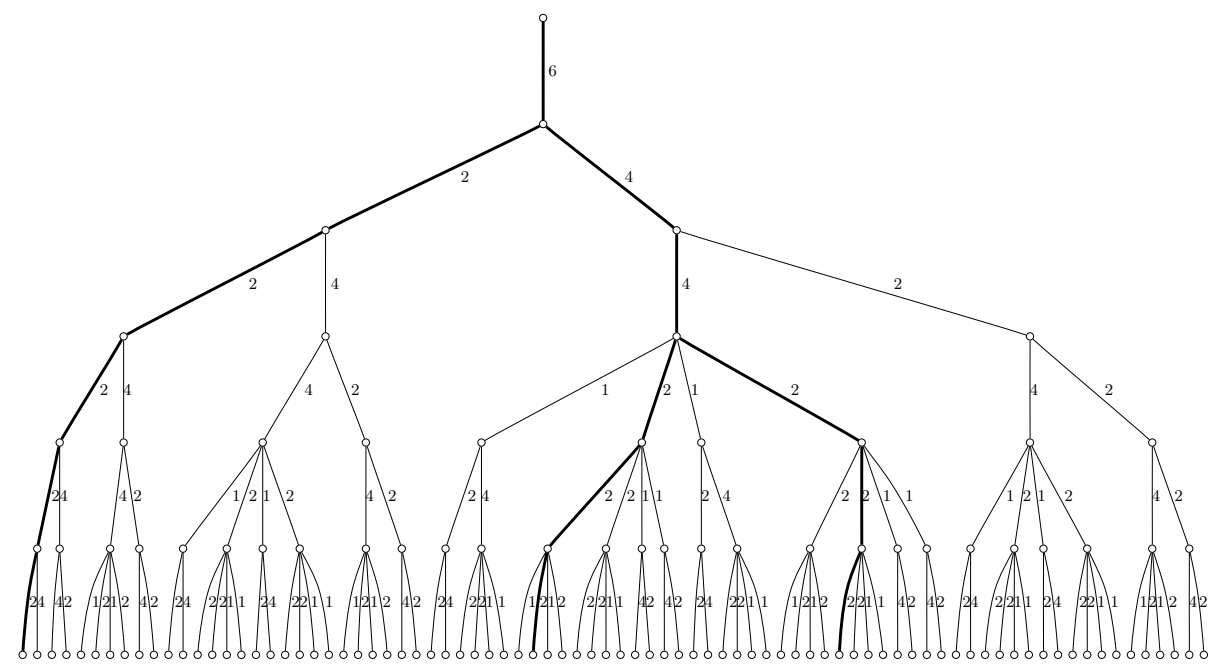

Fig. 4: The labeled orbit tree $\mathfrak{t}(\mathcal{J})$ (up to level 6) with $\mathcal{J}$ defined on Figure 0 (the thickened edges emphasize the 1-self-liftable paths defined below in Def. 177).

Since the orbit trees are rooted, we choose the classical orientation where the root is the higher vertex and the tree grows from top to bottom. A path is a (possibly infinite) sequence of adjacent edges without backtracking. The initial vertex of an edge $e$ is denoted by $T(e)$ and its terminal vertex by $\perp(e)$; by extension, the initial vertex of a non-empty path $\mathbf{e}$ is denoted by $T(\mathbf{e})$ and its terminal vertex by $\perp$ (e) whenever the path is finite. The label of a (possibly infinite) path is the ordered sequence of labels of its edges.

Definition 14. In a tree, a (possibly infinite) path $\mathbf{e}$ is said to be initial if $\top(\mathbf{e})$ is the root of the tree.

Definition 15. Let $\mathcal{A}$ be a reversible Mealy automaton and let $e, f$ be two edges in the orbit tree $\mathrm{t}(\mathcal{A})$. We say that $e$ is liftable to $f$ if each word of $\perp(e)$ admits some word of $\perp(f)$ as a suffix.

One can notice that this condition is not as strong as it seems: 
Lemma 16. Let $\mathcal{A}$ be a reversible Mealy automaton and let e, $f$ be two edges in the orbit tree $\mathfrak{t}(\mathcal{A})$. If there exists a word of $\perp(e)$ which admits a word of $\perp(f)$ as suffix, then e is liftable to $f$.

Proof. Assume $\mathbf{u v} \in \perp(e)$ with $\mathbf{v} \in \perp(f)$. By reversibility, for any word $\mathbf{w}$ in the connected component $\perp(e)$, there exists $\mathbf{s} \in \Sigma^{*}$ satisfying $\mathbf{w}=\delta_{\mathbf{s}}(\mathbf{u v})$, which can also be written $\mathbf{w}=\delta_{\mathbf{s}}(\mathbf{u}) \delta_{\mathbf{t}}(\mathbf{v})$ with $\mathbf{t}=\rho_{\mathbf{u}}(\mathbf{s})$. Hence the suffix $\delta_{\mathbf{t}}(\mathbf{v})$ of $\mathbf{w}$ belongs to the connected component $\perp(f)$ of $\mathbf{v}$.

Definition 17. Let $\mathcal{A}$ be a reversible Mealy automaton and let $\mathbf{e}$ be a (possibly infinite) initial path in the orbit tree $\mathfrak{t}(\mathcal{A})$. We say that $\mathbf{e}=e_{0} e_{1} \cdots$ is 1 -selfliftable whenever every edge $e_{i+1}$ is liftable to its predecessor $e_{i}$, for $i \geq 0$.

This important notion generalizes that of an e-liftable path used in 20] where the liftability is required with respect to a uniquely specified edge $e$.

Using thickened edges, Figure 4 highlights each of the 1-self-liftable paths in the orbit tree $\mathfrak{t}(\mathcal{J})$, where the Mealy automaton $\mathcal{J}$ is displayed on Figure 2 ,

Definition 18. Let $\mathcal{A}$ be a reversible Mealy automaton with stateset $Q$. The path of a word $\mathbf{u} \in Q^{*} \cup Q^{\omega}$ is the unique initial path in $\mathfrak{t}(\mathcal{A})$ going from the root through the connected components of the prefixes of $\mathbf{u}$.

Lemma 19. Let $\mathcal{A}$ be a reversible Mealy automaton. For any state $x$ of $\mathcal{A}$, the path of $x^{\omega}$ in $\mathfrak{t}(\mathcal{A})$ is 1-self-liftable.

Proof. By Lemma 16, $x^{n}$ being a suffix of $x^{n+1}$, such a path is 1-self-liftable.

Lemma 19 guarantees the existence of 1-self-liftable paths in any orbit tree.

\section{Main Result}

Assume that $\mathcal{A}$ is an invertible reversible Mealy automaton without bireversible component. Our aim is to prove that every element of $\langle\mathcal{A}\rangle_{+}$has infinite order. We first prove this property for the states of $\mathcal{A}$, whenever $\mathcal{A}$ is connected, by looking at some 1-self-liftable paths in $\mathfrak{t}(\mathcal{A})$ (defined in Section 4 ). Then we extend it to arbitrary elements of $\langle\mathcal{A}\rangle_{+}$by using the properties of products of Mealy automata (established in Section 3).

Proposition 20. Let $\mathcal{A}$ be some connected invertible reversible non-bireversible Mealy automaton. A 1-self-liftable path in $\mathfrak{t}(\mathcal{A})$ cannot contain an edge labeled by 1.

Proof. Let $\mathcal{A}=(Q, \Sigma, \delta, \rho)$, e be a 1-self-liftable path in $\mathfrak{t}(\mathcal{A})$ and $e$ be an edge of e. Let $T$ (resp. $L$ ) denote the set of states of $T(e)$ (resp. of $\perp(e)$ ). For any word $\mathbf{w}, L_{\mathbf{w}}=\{\mathbf{u} \mid \mathbf{w} \mathbf{u} \in L\}$ is the left quotient of $L$ by $\mathbf{w}$ (see for instance [26]). As $\mathcal{A}$ is connected and reversible, according to Lemma $[6$, for any $x \in Q$, the left quotient $L_{x}$ is non-empty. Hence we have

$$
L=\bigsqcup_{x \in Q} x L_{x} \quad \text { (disjoint union). }
$$


The hypotheses that the path $\mathbf{e}$ is 1 -self-liftable and that $\mathcal{A}$ is invertible yield

$$
T=\bigcup_{x \in Q} L_{x}
$$

Indeed, let $x \mathbf{u} \in L$ with $x \in Q$ (and $\mathbf{u} \in T$ by 1-self-liftability) and let $\mathbf{v} \in T$. By reversibility, there exists $\mathbf{s} \in \Sigma^{*}$ verifying $\delta_{\mathbf{s}}(\mathbf{u})=\mathbf{v}$. Now, by invertibility, there exists $\mathbf{t} \in \Sigma^{*}$ with $\rho_{x}(\mathbf{t})=\mathbf{s}$ :

$$
\begin{aligned}
& x \underset{\vee}{\stackrel{\mathrm{t}}{\longrightarrow}} \delta_{\mathrm{t}}(x)=x^{\prime} \\
& \mathbf{u} \underset{\rho_{\mathbf{u}}(\mathbf{s}) .}{\stackrel{\mathbf{s}}{\longrightarrow}} \delta_{\mathbf{s}}(\mathbf{u})=\mathbf{v}
\end{aligned}
$$

Therefore, $\mathbf{v}$ is a suffix of $\delta_{\mathbf{t}}(x \mathbf{u})$, hence $\mathbf{v} \in L_{x^{\prime}}$ for $x^{\prime}=\delta_{\mathbf{t}}(x) \in Q$.

Since $\mathcal{A}$ is not coreversible, there exist $y \neq y^{\prime}, z \in Q$ and $i, j, k \in \Sigma$ satisfying

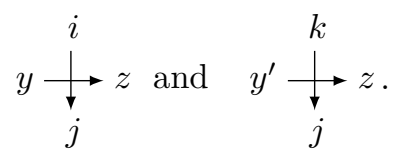

So, in the connected component $\perp(e)$, we have

$$
\delta_{i}\left(y L_{y}\right)=z L_{z} \quad \text { and } \quad \delta_{k}\left(y^{\prime} L_{y^{\prime}}\right)=z L_{z} .
$$

From reversibility of $\mathcal{A}, \delta_{j}$ is injective and we deduce $L_{y}=L_{y^{\prime}}$. Therefore the union $T=\bigcup_{x \in Q} L_{x}$ is not disjoint and we find $|T|<|L|$ which implies that the label of $e$ is greater than 1 .

An easy but interesting first consequence is the following.

Corollary 21. A connected 3-state invertible reversible non-bireversible Mealy automaton generates a free semigroup.

Proof. We deduce from Proposition 20 that any connected 3-state invertible reversible non-bireversible Mealy automaton sees its dual to be spherically transitive and the result follows from [18, Proposition 14].

Let us go back to our main purpose.

Proposition 22. Let $\mathcal{A}$ be some connected invertible reversible non-bireversible Mealy automaton. Then any state of $\mathcal{A}$ induces an action of infinite order.

Proof. Let $x$ be a state of $\mathcal{A}$. The path of $x^{\omega}$ is 1 -self-liftable by Lemma 19, So by Proposition 20, this path has no edge labeled with 1, which means that the sizes of the connected components of $\left(x^{n}\right)_{n \in \mathbb{N}}$ are unbounded. By Proposition 11, the action induced by $x$ has infinite order. 
We can now state our main result by extending Proposition 22 .

Theorem 23. Any invertible reversible Mealy automaton without bireversible component generates a torsion-free semigroup.

Proof. Let $\mathcal{A}$ be an invertible reversible Mealy automaton without bireversible component with stateset $Q$. Let $\mathbf{u} \in Q^{+}$and let $\mathcal{C}$ its connected component in $\mathfrak{t}(\mathcal{A})$. From Corollary $8, \mathcal{C}$ is a connected invertible reversible non-bireversible Mealy automaton with $\mathbf{u}$ as a state. Hence by Proposition 22, $\mathbf{u}$ induces an action of infinite order.

Note that Theorem 23 cannot provide extra information on the torsion-freeness of the generated group. Take for instance the Mealy automaton $\mathcal{L}$ of Figure 1 (left): the action induced by $y x^{-1}$ has order 2 . However, Theorem 23] ensures that an invertible reversible Mealy automaton without bireversible component cannot generate an infinite Burnside group (see 24] for background on the Burnside problem).

All these results and constructions emphasize the relevance of the reversibility property and question us further on those (semi)groups structures generated by bireversible automata that, despite the tightness of the hypothesis on them, reveal more complex to study.

\section{Acknowledgments}

The authors thank an anonymous referee, whose relevant comments improved the paper.

\section{References}

1. Akhavi, A., Klimann, I., Lombardy, S., Mairesse, J., Picantin, M.: On the finiteness problem for automaton (semi)groups. Internat. J. Algebra Comput. 22(6), 26p. (2012)

2. Alešin, S.V.: Finite automata and the Burnside problem for periodic groups. Mat. Zametki 11, 319-328 (1972)

3. Antonenko, A.S.: On transition functions of Mealy automata of finite growth. Matematychni Studii. 29(1), 3-17 (2008)

4. Antonenko, A.S., Berkovich, E.L.: Groups and semigroups defined by some classes of Mealy automata. Acta Cybernetica 18(1), 23-46 (2007)

5. Bartholdi, L., Kaimanovich, V.A., Nekrashevych, V.V.: On amenability of automata groups. Duke Math. J. 154(3), 575-598 (2010)

6. Bartholdi, L., Reznykov, I.I., Sushchanskiǔ, V.I.: The smallest Mealy automaton of intermediate growth. J. Algebra 295(2), 387-414 (2006)

7. Bartholdi, L., Silva, P.V.: Groups defined by automata (2010), chapter 24 of the handbook AutoMathA, ArXiv:cs.FL/1012.1531

8. Baumslag, G., Boone, W.W., Neumann, B.H.: Some unsolvable problems about elements and subgroups of groups. Math. Scand. 7, 191-201 (1959) 
9. Bondarenko, I., Grigorchuk, R.I., Kravchenko, R., Muntyan, Y., Nekrashevych, V., Savchuk, D., Sunić, Z.: On classification of groups generated by 3-state automata over a 2-letter alphabet. Algebra Discrete Math. (1), 1-163 (2008)

10. Bondarenko, I.V., Bondarenko, N.V., Sidki, S.N., Zapata, F.R.: On the conjugacy problem for finite-state automorphisms of regular rooted trees. Groups Geom. Dyn. 7(2), 323-355 (2013), with an appendix by Raphaël M. Jungers

11. Cain, A.J.: Automaton semigroups. Theor. Comput. Sci. 410, 5022-5038 (2009)

12. Eilenberg, S.: Automata, languages, and machines. Vol. A. Academic Press [A subsidiary of Harcourt Brace Jovanovich, Publishers], New York (1974)

13. Gawron, P.W., Nekrashevych, V.V., Sushchansky, V.I.: Conjugation in tree automorphism groups. Internat. J. Algebra Comput. 11(5), 529-547 (2001)

14. Gillibert, P.: The finiteness problem for automaton semigroups is undecidable. Internat. J. Algebra Comput. 24(1), 1-9 (2014)

15. Grigorchuk, R.I.: On Burnside's problem on periodic groups. Funktsional. Anal. i Prilozhen. 14(1), 53-54 (1980)

16. Grigorchuk, R.I.: On the Milnor problem of group growth. Dokl. Akad. Nauk SSSR 271(1), 30-33 (1983)

17. Grigorchuk, R.I., Nekrashevich, V.V., Sushchanskiı̆, V.I.: Automata, dynamical systems, and groups. Tr. Mat. Inst. Steklova 231, 134-214 (2000)

18. Klimann, I.: The finiteness of a group generated by a 2-letter invertible-reversible Mealy automaton is decidable. In: Proc. 30th STACS. LIPIcs, vol. 20, pp. 502-513 (2013)

19. Klimann, I., Mairesse, J., Picantin, M.: Implementing computations in automaton (semi)groups. In: Proc. 30th CIAA. LNCS, vol. 7381, pp. 240-252 (2012)

20. Klimann, I., Picantin, M., Savchuk, D.: A connected 3-state reversible Mealy automaton cannot generate an infinite Burnside group (2014), arXiv:1409.6142

21. Macedonska, O., Nekrashevych, V.V., Sushchansky, V.I.: Commensurators of groups and reversible automata. Dopov. Nats. Akad. Nauk Ukr., Mat. Pryr. Tekh. Nauky (12), 36-39 (2000)

22. Maltcev, V.: Cayley automaton semigroups. Internat. J. Algebra Comput. 19(1), 79-95 (2009)

23. Mintz, A.: On the Cayley semigroup of a finite aperiodic semigroup. Internat. J. Algebra Comput. 19(6), 723-746 (2009)

24. Nekrashevych, V.: Self-similar groups, Mathematical Surveys and Monographs, vol. 117. American Mathematical Society, Providence, RI (2005)

25. Russyev, A.: Finite groups as groups of automata with no cycles with exit. Algebra and Discrete Mathematics 9(1), 86-102 (2010)

26. Sakarovitch, J.: Elements of Automata Theory. Cambridge University Press (2009)

27. Sidki, S.N.: Automorphisms of one-rooted trees: growth, circuit structure, and acyclicity. J. Math. Sci. (New York) 100(1), 1925-1943 (2000), algebra, 12

28. Silva, P.V., Steinberg, B.: On a class of automata groups generalizing lamplighter groups. Internat. J. Algebra Comput. 15(5-6), 1213-1234 (2005) 